\title{
Arithmetic in the adult deaf signing brain
}

Josefine Andin, Åsa Elwér and Elina Maki-Torkko

The self-archived postprint version of this journal article is available at Linköping University Institutional Repository (DiVA):

http://urn.kb.se/resolve?urn=urn:nbn:se:liu:diva-162723

N.B.: When citing this work, cite the original publication.

Andin, J., Elwér, Å., Maki-Torkko, E., (2019), Arithmetic in the adult deaf signing brain, Journal of Neuroscience Research. https://doi.org/10.1002/jnr.24569

Original publication available at:

https://doi.org/10.1002/jnr.24569

Copyright: Wiley (12 months)

http://eu.wiley.com/WileyCDA/ 


\section{Arithmetic in the adult deaf signing brain}

Josefine Andin ${ }^{1,2}$, Åsa Elwér ${ }^{1}$, Elina Mäki-Torkko ${ }^{2,3}$

1. Department of Behavioural Sciences and Learning, Linköping University, Linköping, Sweden

2. Swedish Institute for Disability Research

3. Faculty of Medicine and Health, Örebro University, Örebro, Sweden

Running head: Arithmetic in the deaf signing brain

Associate editor: Dr. Eric Prager and Dr. Jeremy Hogeveen

Keywords: deafness, sign language, arithmetic, functional magnetic resonance imaging

Grant: Swedish research council, 2016-02337

Corresponding author: Josefine Andin, Josefine.andin@liu.se, +46 (0)13282165 


\begin{abstract}
We have previously shown that deaf signers recruit partially different brain regions during simple arithmetic compared to a group of hearing non-signers, despite similar performance. Specifically, hearing individuals show more widespread activation in brain areas that have been related to the verbal system of numerical processing, i.e. left angular and inferior frontal gyrus, whereas deaf individuals engaged brain areas that have been related to the quantity system of numerical processing, i.e. right horizontal intraparietal sulcus. This indicates that compared to hearing nonsigners, deaf signers can successfully make use of processes located to partially different brain areas during simple arithmetic. In this study, which is a conceptual replication and extension of the abovepresented study, the main aim is to understand similarities and differences in neural correlates supporting arithmetic in deaf compared to hearing individuals. The primary objective is to investigate the role of the right horizontal intraparietal gyrus, the left inferior frontal gyrus, the hippocampus, and the left angular gyrus during simple and difficult arithmetic and how these regions are connected to each other. A second objective is to explore what other brain regions support arithmetic in deaf signers. Up to 34 adult deaf signers and the same amount of hearing non-signers will be enrolled in an fMRI-study of simple as well as difficult subtraction and multiplication. Brain imaging data will be analyzed using whole-brain analysis, region of interest analysis and connectivity analysis. This is the first study to investigate neural underpinnings of arithmetic of different difficulties in deaf individuals.
\end{abstract}

\title{
Significance statement
}

The purpose of this study is to understand similarities and differences in neural correlates supporting arithmetic in adult deaf signers and hearing non-signers. We expect to find deaf signers to rely on quantity processes to a larger extent and verbal processes to a lesser extent compared to hearing non-signers. 


\section{Introduction}

International research has shown that deaf signers lag several years behind hearing peers in mathematics in general (Pagliaro, 2010). However, this is not necessarily the case for all mathematical domains. In a recent study at our lab, Swedish deaf adults were shown to perform on par with hearing peers on simple multiplication and simple subtraction and yet show differences in the recruitment of classical language and magnitude processing brain areas (Andin, Fransson, Dahlström, Rönnberg, \& Rudner, 2019). Specifically, hearing individuals show more widespread activation in brain areas that have been related to verbal processing of arithmetic facts in the left inferior frontal gyrus, whereas deaf individuals engaged brain areas that have been related to language-independent magnitude processing in the right intraparietal sulcus when performing simple arithmetic. This indicates that, compared to hearing non-signers, deaf signers can successfully make use of processes located to partially different brain areas during simple arithmetic. The main aim of the current study is to further our understanding of similarities and differences in neural correlates supporting simple and difficult arithmetic in deaf signers compared to hearing non-signers. As such, this study is a conceptual replication with extension of Andin et al. (2019).

Signed languages are visual, natural and complete languages in the visuospatial domain (Sandler \& Lillo-Martin, 2006) that support language development in much the same way as spoken languages do (Mayberry \& Lock, 2003). In 1983, a new curriculum for deaf education was introduced in Sweden and since then all deaf children and their families are offered sign language courses and support for the child in preschool settings from the age of 1 ( $(\mathrm{Gr} \mathrm{80,1983).} \mathrm{During} \mathrm{the} \mathrm{80s,} \mathrm{90s,} \mathrm{and} \mathrm{00s,} \mathrm{before}$ the introduction of cochlear implants, almost every deaf child in Sweden attended a deaf school during their formal schooling from preschool to high school. This means that they have followed a bilingual curriculum where Swedish sign language has been the main mode of communication and written Swedish is considered a second language (e.g. Bagga-Gupta, 2004). This has led to favorable linguistic development for Swedish deaf children of both deaf and hearing parents born in the last three decades of the $20^{\text {th }}$ century (Roos, 2006). Therefore, the young Swedish adult deaf signers constitute a unique population for whom sign language learning has been optimized (Bagga-Gupta, 2004). This is in contrast to many other deaf signing populations in countries where oral education of deaf children is still common and where there is a larger variability in preferred language in the deaf population. However, with the contemporary introduction of cochlear implants, the scenery has changed as most children who are born deaf will have access to the auditive domain, and therefore sign language may become used to a lesser extent during childhood. Therefore, it is important to take the opportunity to investigate language modality dependent and independent processes in this deaf young adult population for whom sign language learning has been optimized. This is of both theoretical and practical importance. From a theoretical point of view, this study will broaden knowledge of how cognitive processes are supported by the brain. The present study will also be of importance for future studies on other populations since it generates knowledge about cognitive functions in individuals that are solely dependent on the visual modality for communication. Such knowledge will be important when formulating hypotheses about cognitive functions in individuals that rely on both the visual and the auditory modality, especially individuals with cochlear implants. Because the use of imaging techniques in the cochlear implanted population is limited, it is important to explore the signing brain using e.g. functional magnetic resonance imaging (fMRI) to generate testable hypotheses that can be explored with less elaborate imaging techniques such as functional near-infrared spectroscopy (fNIRS), which is compatible with cochlear implants. Furthermore, there will always be individuals for whom cochlear implants are not an option, e.g. due to missing cochleae, medical or financial reasons. Therefore, knowledge generated by this study will be valuable to inform teaching strategies for this small group. Furthermore, the present study could also add 
valuable information in relation to other groups with mathematical difficulties, such as developmental as well as acquired dyscalculia, where it might be difficult to disentangle linguistic and mathematical aspects.

Arithmetic concerns the basic operations of numbers, i.e. addition, subtraction, multiplication, and division. Evidence suggests that both verbal and quantity competencies are involved when engaging with arithmetic operations (Dehaene, Piazza, Pinel, \& Cohen, 2003; Lee \& Kang, 2002; Zhou et al., 2018). Quantity competences involve magnitude manipulations along a mental analog number line and verbal competencies come into play when pre-learned facts are retrieved from long term memory. These two competencies are engaged to different degrees depending on the operation at hand. In this sense, the operations can be considered to represent a continuum with multiplication and subtraction representing the extremes, while addition and division are placed in between the two. Multiplication primarily taxes verbal competence as the multiplication tables typically are learned by rote learning and can be retrieved by arithmetic fact retrieval. Subtraction, on the other hand, mainly taxes quantity competences as subtraction is less likely to be learned by rote learning and instead requires manipulation along the mental number line. The exception being simple subtraction tasks, where retrieval strategies can also be used (e.g. Andin et al., 2019; Simon, Mangin, Cohen, Le Bihan, \& Dehaene, 2002). Several studies have found that the verbal and the quantity competencies are supported by partially different neural correlates (e.g. Dehaene et al., 2003; Prado et al., 2011). The rationale for including multiplication and subtraction in most neuroimaging studies (including the present) is to target these two competencies.

Perhaps the most influential model of number processing, the triple-code model, proposes three different systems for number processing of which the verbal and quantity system are two, the third being the attentional system (Dehaene et al., 2003). Although recent brain imaging research has challenged the validity of the model, the verbal and the quantity systems are consistently implicated in arithmetic processing (for review see Arsalidou, Pawliw-Levac, Sadeghi, \& Pascual-Leone, 2018; Arsalidou \& Taylor, 2011).

The verbal system includes verbal representations of numbers and is especially involved in arithmetic fact retrieval. Deaf individuals have been found to perform worse than hearing individuals on a number of tasks related to the verbal system, e.g. relational statements (Kelly, Lang, Mousley, \& Davis, 2003; Serrano Pau, 1995), arithmetic word problems that require reading (Hyde, Zevenbergen, \& Power, 2003), fractions (Titus, 1995) and multiplication (Andin, Ronnberg, \& Rudner, 2014; Nunes et al., 2009). According to the original triple-code model the left angular gyrus was suggested to be the main region for the verbal system (Dehaene et al., 2003), which is further supported by several other studies (e.g. Grabner et al., 2009; Grabner, Reishofer, Koschutnig, \& Ebner, 2011; Price \& Ansari, 2011). In the left angular gyrus, activation has been found for tasks of exact compared to approximate calculation, small compared to large numbers and multiplication compared to subtraction (for a review see Dehaene et al., 2003). Activation has also been reported to decrease with the increased complexity of the task at hand (Artemenko et al., 2019) and increase with mathematical competence (Grabner et al., 2007). However, several recent studies have failed to find activation for arithmetic tasks in this region (Grabner, Ansari, Koschutnig, Reishofer, \& Ebner, 2013; Wu et al., 2009). In a recent study, when using the whole left angular gyrus as a region of interest it was deactivated for both hearing and deaf individuals for simple subtraction and multiplication compared to a baseline task (Andin et al., 2019). However, the posterior and the anterior portion of left angular gyrus show distinct differences in terms of both structural and functional connectivity (Uddin et al., 2010). The posterior part of the left angular gyrus constitutes the lateral parietal node of the default mode network, meaning that this region is activated during rest and deactivated 
during tasks that require attention to shift from default mode to task. Accordingly, the engagement of the posterior left angular gyrus during arithmetic processing might be related to difficulty modulations rather than to arithmetic tasks per se (Wu et al., 2009). The anterior left angular gyrus, on the other hand, does not seem to be as strongly connected to the default mode network and show less or no deactivation during arithmetic tasks. Uddin et al. (2010) argue that dividing left angular gyrus into two different regions is important for understanding how these regions are engaged during numerical cognition. Despite inconsistency between studies, it is uncontested that left angular gyrus is of importance for arithmetic processing. However, another line of research shows that, although the left angular gyrus is important during arithmetic fact retrieval, its role is merely to mediate and allocate attention during retrieval (Bloechle et al., 2016; Klein, Willmes, Bieck, Bloechle, \& Moeller, 2019). Instead, there are several studies showing that the region responsible for the encoding and retrieval of arithmetic facts is the hippocampus (Bloechle et al., 2016; Klein et al., 2016). This notion is supported by analyses of functional connectivity between the hippocampus and the left angular gyrus, where Klein et al. (2016) has shown that the angular gyrus and the hippocampus are functionally connected for fact retrieval tasks, but not for magnitude processing in a hearing population. If the role of the angular gyrus is to mediate hippocampal function it is likely that this connection is less apparent in deaf individuals. However, as hippocampus is a general hub for memory formation (e.g. Eichenbaum, 2004) and deaf individuals do not have any specific memory deficit, it is possible that the hippocampus may be connected to other regions, such as the right intraparietal sulcus, which would indicate a different route to fact retrieval via magnitude processing in deaf signers.

A further extension of the verbal system, from left angular gyrus towards larger parts of the leftlateralized perisylvian network has been suggested by several researchers (Arsalidou \& Taylor, 2011; Fedorenko, Duncan, \& Kanwisher, 2012; Skagenholt, Träff, Västfjäll, \& Skagerlund, 2018). Especially, the left inferior frontal gyrus, which is generally activated during verbal tasks, seems like a promising region for arithmetic reasoning. This region has been found to be activated during size comparison task for both digits and number words compared to letters (Skagenholt et al., 2018), for difficult versus simple arithmetic tasks (Fedorenko et al., 2012), two-digit versus one-digit multiplication problems (Soltanlou et al., 2017) and for carrying and borrowing in high versus low performing individuals (Artemenko, Soltanlou, Dresler, Ehlis, \& Nuerk, 2018). Recent studies have further shown that resting-state functional connectivity between the left inferior frontal gyrus and the angular gyrus is stronger for individuals that are proficient in arithmetic (Skagerlund et al., 2019). Because deaf individuals seem to show less activation in the verbal system during arithmetic, we expect this connection to be less apparent in deaf compared to hearing individuals.

The quantity system is primarily involved in magnitude manipulation along the analog mental number line. For tasks associated to this system there is no evidence of poorer performance for deaf individuals: Deaf and hearing children perform at similar levels on basic competencies such as subitizing (Bull, Blatto-Vallee, \& Fabich, 2006), magnitude processing (Bull et al., 2006), number comparisons (Bull, Marschark, \& Blatto-Vallee, 2005) and deaf adults perform on par with hearing adults on subtraction (Andin et al., 2014). The quantity system was originally located to the bilateral horizontal portion of the intraparietal sulcus (Dehaene et al., 2003). This region has been shown to be activated more for subtraction than multiplication, more for approximate than exact calculation and more for number words compared to other types of words. Thus, it has been suggested that this region is a primary neural correlate for magnitude processing (Skagenholt et al., 2018; Sokolowski, Fias, Mousa, \& Ansari, 2017). Connectivity studies have suggested that connectivity between the right intraparietal sulcus and several different brain regions is modulated by arithmetic competence (Jolles et al., 2016; Rosenberg-Lee et al., 2015; Skagerlund et al., 2019). Skagerlund et al. (2019) 
showed that individuals with high arithmetic proficiency had stronger connectivity between the right intraparietal sulcus and the left frontal regions as well as the left supramarginal gyrus, whereas connection to right frontal regions was associated with poorer arithmetic competence. For children with developmental dyscalculia, Jolles et al. (2016) and Rosenberg-Lee et al. (2015) found the right intraparietal sulcus to be hyper-connected with several other regions, including bilateral angular gyrus, leading to the suggestion that these children have fundamental differences in their parietal organization compared to normally developing children. Although both deaf individuals and individuals with developmental dyscalculia perform under par in arithmetic we do not know if the basis for the problems share mechanism for the two groups. In the present study, we will investigate how connectivity from this region differ between deaf and hearing individuals.

The neuronal correlates underlying number processing in deaf individuals are poorly understood. To the best of our knowledge, there are only three imaging studies on number processing in deaf signers (Andin et al., 2019; Andin, Fransson, Ronnberg, \& Rudner, 2018; Masataka, Ohnishi, Imabayashi, Hirakata, \& Matsuda, 2006). Masataka et al. (2006) showed that neural systems similar to those found for calculation in hearing individuals are activated when deaf individuals learned the signed numerals of another sign language than their own. In a number and letter order task in Andin et al. (2018), there was a tendency towards stronger activation of the right horizontal intraparietal sulcus in deaf compared to hearing individuals. In the only study so far investigating neural correlates of arithmetic processing in deaf signers, the results showed that the right horizontal intraparietal sulcus was significantly recruited for both deaf and hearing individuals during subtraction, but only for deaf individuals during simple multiplication (Andin et al., 2019). The activation differed significantly between groups for simple multiplication, whereas there were no group differences for simple subtraction. We also showed that while both deaf and hearing individuals show significant activation for multiplication in the left inferior frontal gyrus, only hearing individuals show significant activation for subtraction (between-group analyses were not significant in either comparison). Importantly, there was no significant difference in performance in either response time or accuracy for subtraction or multiplication. As mentioned above, the left angular gyrus was not found to be significantly activated during simple arithmetic for either group (Andin et al., 2019). However, it should be noted that the stimuli material was designed to be used for different tasks including digit and letter order (Andin et al., 2018) and phonology (Andin et al., 2019). That stimulus material required a high portion of working memory and other cognitive competences. In the present study, will we use stimuli consisting of pure arithmetic tasks to be able to investigate arithmetic processes in a more direct way.

In domains other than arithmetic, it has been shown that deaf individuals have stronger connectivity to and from the superior and middle temporal cortices for task versus no task (Malaia, Talavage, \& Wilbur, 2014), during reading (Hirshorn, Dye, Hauser, Supalla, \& Bavelier, 2014) and during resting state (Cardin et al., 2018). Whether connectivity between regions involved in arithmetic and temporal cortex differs between deaf and hearing individuals is unknown. Further, as temporal regions are found to be involved in linguistic and cognitive tasks to a higher degree for deaf compared to hearing individuals (Cardin et al., 2018; Twomey, Waters, Price, Evans, \& MacSweeney, 2017), it is possible that temporal regions have a role in arithmetic processing for deaf individuals, which would show up as connections between the right intraparietal sulcus and temporal regions as well as in activation of temporal regions in the whole-brain analysis.

Combining results from behavioral and imaging studies suggest that when engaging in arithmetic tasks deaf signers successfully make use of different strategies and different neuronal regions compared to hearing non-signers. These results call for further exploration of the hypotheses that 
deaf signers recruit regions related to quantity processing for simple subtraction and regions related to verbal and quantity processing during simple multiplication, whereas hearing non-signers recruit regions related to verbal processing during simple multiplication and regions related to both verbal and quantity processing during simple subtraction. There are no studies on neural correlates of difficult arithmetic in deaf populations. However, for hearing individuals it is expected that they will recruit more of the quantitative system for multiplication beyond the rote-learned multiplication tables which are not retrieved by arithmetic fact retrieval, but possibly through approximation strategies (Dehaene, 1992; Dehaene et al., 2003; Ganor-Stern, 2016; Soltanlou et al., 2017). Further, several studies have shown that the triple code model is a reliable model of numerical cognition, although with some changes, for hearing individuals. In this study, we will investigate the universality of the model in the deaf population that uses a different language modality, i.e. visuospatial rather than auditory language. See figure 1 for an overview of these hypotheses.
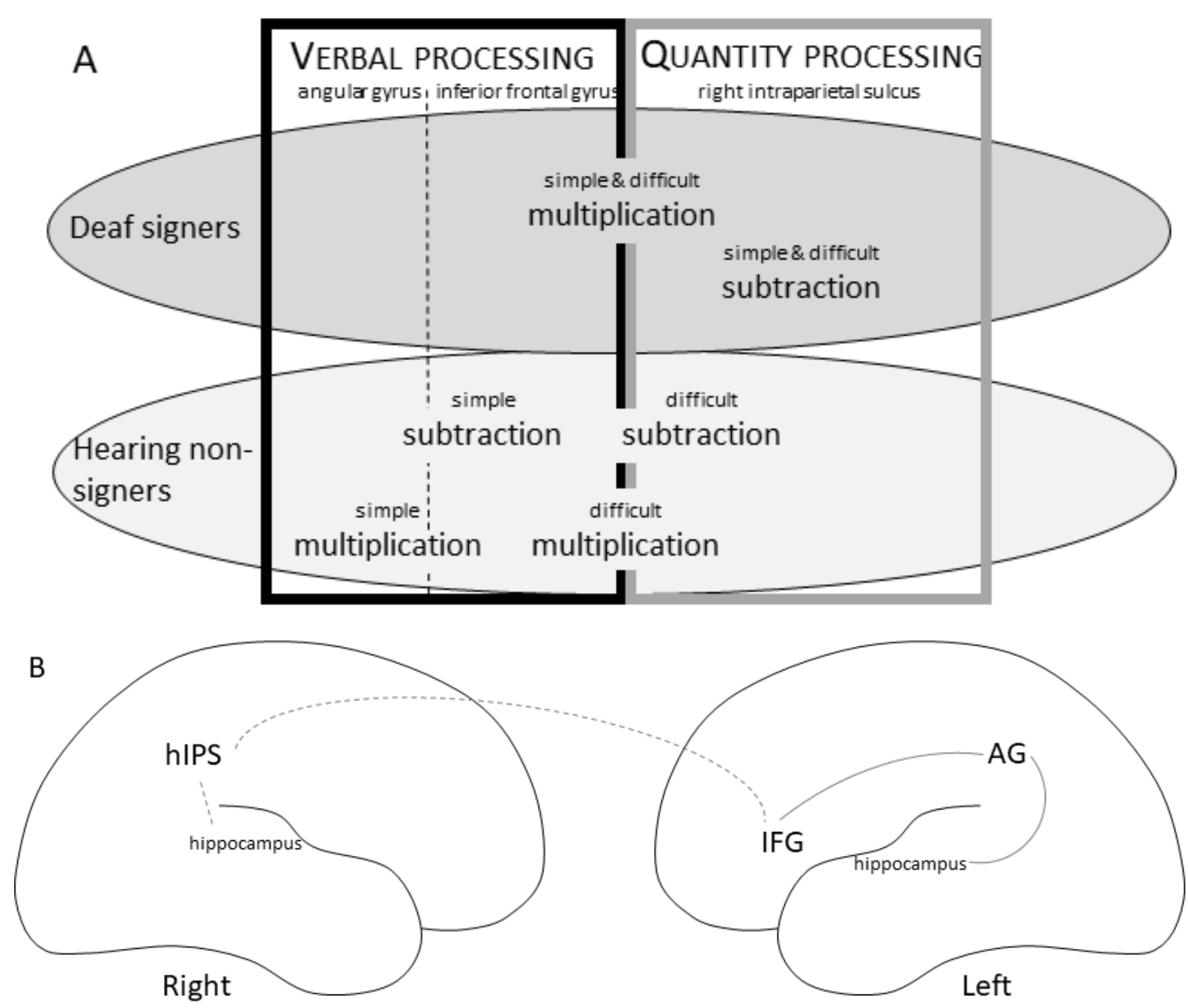

Figure 1. Overview of predictions tested in the present study. A) Predictions primarily derived from Andin et al.(2019) suggest that deaf signers recruit regions related to quantity processing for simple subtraction (activation in right horizontal intraparietal sulcus but not in left inferior frontal gyrus and left angular gyrus/hippocampus) and regions related to both verbal and quantity processing during simple multiplication (activation in both right horizontal intraparietal sulcus, left inferior frontal gyrus but probably not in left angular gyrus/hippocampus), whereas hearing non-signers recruit regions related to verbal processing during simple multiplication (activation of left inferior frontal gyrus and left angular gyrus/hippocampus but not right horizontal intraparietal sulcus) and regions related to both verbal and quantity processing during 
simple subtraction (activation of both left inferior frontal gyrus, left angular gyrus/hippocampus and right horizontal intraparietal sulcus). Difficult multiplication will recruit both systems for both groups, whereas difficult subtraction will recruit mainly quantity processing for both groups. B) Task-based connectivity analyses will show connectivity between angular gyrus, inferior frontal gyrus, and hippocampus for hearing individuals (grey lines), whereas for deaf individuals there will be connections between the right intraparietal sulcus and inferior frontal gyrus and possibly hippocampus (dotted grey line). hIPS horizontal portion of the intraparietal sulcus, AG angular gyrus, IFG inferior frontal gyrus.

Aim

This study is a conceptual replication and extension of Andin et al. (2019) with more power. The conceptual replication includes simple multiplication and subtraction, but with a stimulus material more suited for analyses of arithmetic processes and the extension includes difficult multiplication and subtraction as well as connectivity analyses. The overarching aim of the present study is to understand similarities and differences in neural correlates supporting arithmetic in adult deaf signers and hearing non-signers. The primary objective is to investigate the role of the right horizontal intraparietal gyrus, the left inferior frontal gyrus, the left angular gyrus and, the hippocampus during simple and difficult arithmetic. In detail, we predict that:

1) Deaf signers will show activation in:

a) the right horizontal intraparietal sulcus for both simple and difficult subtraction and multiplication,

b) the left inferior frontal gyrus and left angular gyrus for multiplication,

c) the hippocampus for simple multiplication and subtraction.

2) Hearing non-signers will show activation in:

a) the right horizontal intraparietal sulcus for difficult subtraction and difficult multiplication,

b) the left inferior frontal gyrus and left angular gyrus for multiplication and subtraction,

c) the hippocampus for simple multiplication and subtraction.

3) There will be differences between groups such that:

a) deaf signers will show stronger activation compared to hearing non-signers in the right horizontal intraparietal sulcus for simple multiplication,

b) hearing non-signers will show stronger activation compared to deaf signers in the left inferior frontal gyrus and possibly also in the left angular gyrus for subtraction and simple multiplication,

c) hearing non-signers will show stronger activation compared to deaf signers in the hippocampus for multiplication.

4) There will be group differences in how the involved regions are connected. We expect to find connectivity between:

a) the right intraparietal sulcus, the left inferior frontal gyrus and possibly the hippocampus for the deaf group,

b) the left angular gyrus, the left inferior frontal gyrus and, the hippocampus in hearing individuals, whereas such connectivity will be weaker or not found at all for deaf individuals.

The second objective is to explore what other brain regions support arithmetic in deaf signers. We expect to find:

5) mainly similar networks recruited for both groups at whole-brain analyses,

6) superior temporal regions (primary and secondary auditory cortex) to be involved in arithmetic for deaf individuals,

7) stronger connectivity from the arithmetic network to superior temporal regions for deaf signers compared to hearing non-signers. 


\section{Methods}

\section{Power analysis}

To the best of our knowledge, there are two main ways of performing power analyses on fMRI studies. The first is to do a pilot study of the design on a small sample of participants and the second is to use statistical maps from similar studies. In this project, pilot work will be very difficult because the population of deaf signers in Sweden is so small that it will be impossible to find enough participants for both a pilot and the main study. It would be possible to do a pilot study on hearing individuals, but that would not be helpful as we are interested in group differences. The other option is to use statistical maps from a similar study. The only previous fMRI-study on arithmetic in deaf signers is Andin et al. (2019) and in that study, we did not investigate difficult arithmetic tasks. However, as this is the only study available, fMRI power analyses were performed using that study. The fMRIpower software (fmripower.org, Mumford \& Nichols, 2008) was used to estimate the power to detect significant activation within specific regions of interest with effect size given in SD-units (equivalent to the Cohen's D measure). T-maps were used for the power estimations, as this tool does not allow for power calculations of more complex designs. We performed power calculations on each group individually (prediction 1 and 2) using one-sample t-tests (alpha level 0.05) and for group comparisons (prediction 3) using two-sample $t$-tests (alpha level of 0.05).

Related to prediction 1 we will, for the deaf group, have at least $80 \%$ power to:

- detect an effect size of 0.83 SD-units with 11 participants for multiplication in the right intraparietal sulcus

- detect an effect size of 0.67 SD-units with 17 participants for subtraction in the right intraparietal sulcus

- detect and effect size of 0.35 SD-units with 16 participants for multiplication in the left inferior frontal sulcus

Related to prediction 2 we will, for the hearing group, have at least $80 \%$ power to:

- detect an effect size of 0.48 SD-units with 27 participants for multiplication in the left inferior frontal gyrus

- detect an effect size of 0.47 SD-units with 28 participants for subtraction in the left inferior frontal gyrus

Related to prediction 3 we will, for group comparisons, have at least $80 \%$ power to:

- detect an effect size of 0.82 with 18 participants per group in the right intraparietal sulcus for multiplication

- detect an effect size of 0.61 with 34 participants per group in the left inferior frontal gyrus for multiplication

For the other contrasts it was not possible to calculate power due to deactivation (in left angular gyrus and partly for hippocampus) and in some cases, the sample size needed was too high to be reasonable for this study. Concerning the hippocampus, the study, at which the power calculations are based on, did not investigate direct fact retrieval, and as fact retrieval is the proposed action of the hippocampus, it is still possible that we will be able to find effects there. Further, based on these calculations we will not have power to detect effects of subtraction in any region for hearing individuals. This can be a problem, but it should be noted that these calculations are only performed 
for simple multiplication and subtraction. We expect effects of difficult subtraction to result in stronger activations in the right intraparietal sulcus. Based on these estimates and the fact that the main region of interest is the horizontal intraparietal sulcus, we will aim at scanning 34 participants per group. Excluded participants will be replaced.

However, it should be noted that the current research design varies considerably from the design used for the power estimations. The present study is better optimized with fewer conditions (the Andin et al. 2019-study also included other conditions than arithmetic) and more repetitions of each condition. The magnetic field of the MR-scanner in the present study will be higher ( $3 \mathrm{~T}$ compared to $1.5 \mathrm{~T}$ ), which will improve the signal-to-noise ratio and thus lead to higher power. Further, as deaf signers belong to a population that is difficult to recruit and the design in the present study is better optimized to find group differences, we believe that fewer participants will suffice. Based on this and on economical constraints, we will stop collecting data by the end of December 2020 even if the aim at 34 participants per group has not been reached. However, even if we do not reach the goal of 34 participants per group, it should be noted that with a group size of 28 we will have the power to detect all but the effect of multiplication in the left inferior frontal gyrus and with group sizes of 18 we will have enough power to detect effects related to prediction 1 and 3 , which are the two most important predictions in this study. Further, it is common with small groups in fMRI-studies on deaf signers even when they belong to larger sign language populations, such British and American sign language users.

\section{Participants}

Up to 34 deaf early signers will be recruited. Inclusion criteria for the deaf participants will be prelingual deafness, using Swedish sign language as the main mode of communication from early childhood (before age of 3), having been enrolled in bilingual schooling and not having a cochlear implant. The age range will be 18 to approximately 45 years of age (born 1976 or later), allowing us to take advantage of the unique sign language experience of deaf adults that were enrolled in school after the introduction of the bilingual curriculum in 1983 (see table 1 for a summary of inclusion and exclusion criteria). The focus of the study is to investigate arithmetic processing in signing individuals. However, it cannot be precluded that different types of hearing deficits/deafness affects neurobiological networks. Therefore, we will also collect information about audiological diagnosis and language used by parents and siblings and control for this in the analysis, if necessary.

Up to 34 hearing non-signers matched to the deaf early signer group on age, education level, nonverbal intelligence and gender will also be recruited. These participants should be unfamiliar with Swedish sign language and have Swedish as their first language. Non-verbal intelligence will be tested using the visual puzzle subtest from the Wechsler adult intelligence scale, which has been shown to be highly correlated with general intelligence. Participants that perform more than two standard deviations below the mean of the norm group will be excluded from further analyses and replaced.

All participants will be right-handed with no history of neurological or psychological conditions. No sex differences are expected in either group, but we will aim at same-sex distribution in both groups. Instructions will be given orally for hearing and in Swedish sign language for deaf participants (either through a signing scientist/experimenter or by use of a sign language interpreter). Written informed consent will be signed by all participants. Information about the project and the rights of the participants will be given in Swedish, Swedish sign language and in written before consent is signed. The project is approved by the Swedish Ethical Review Authority (Dnr: 2019-00896). Participants will be paid SEK 1000 for their participation (app. \$100). 
Table 1. Summary of inclusion and exclusion criteria for participant inclusion.

\begin{tabular}{|c|c|c|}
\hline & $\begin{array}{l}\text { Common for } \\
\text { both groups }\end{array}$ & $\begin{array}{l}\text { Hearing non- } \\
\text { signers }\end{array}$ \\
\hline \multicolumn{3}{|l|}{ Inclusion criteria } \\
\hline Primary language & Swedish sign language & Spoken Swedish \\
\hline Schooling & Bilingual & Mainstream \\
\hline Hearing status & Deaf & Hearing \\
\hline Age of acquisition for primary language & \multicolumn{2}{|l|}{$<3$ years of age } \\
\hline Handedness & \multicolumn{2}{|l|}{ Right-handed } \\
\hline Year of birth & \multicolumn{2}{|c|}{ After 1975 and before $2001 / 2002^{1}$} \\
\hline \multicolumn{3}{|l|}{ Exclusion criteria } \\
\hline Hearing aids & $\begin{array}{l}\text { Cochlear } \\
\text { implants and } \\
\text { hearing aids }\end{array}$ & $\begin{array}{l}\text { Cochlear } \\
\text { implants and } \\
\text { hearing aids }\end{array}$ \\
\hline Non-verbal intelligence & \multirow{3}{*}{\multicolumn{2}{|c|}{$\begin{array}{l}<2 \text { SD from norm group mean } \\
\text { Neurological or psychiatric conditions } \\
\text { Movement more than } 3 \mathrm{~mm} \text { movement in } x, y, z \text { or } 3 \\
\text { degrees in pitch, yaw, roll, }\end{array}$}} \\
\hline Other conditions & & \\
\hline Scanning & & \\
\hline
\end{tabular}

\section{Procedure}

Upon inclusion in the project, participants will fill in an online form including questions about education, work situation, age and age of acquisition of Swedish sign language. Participants that fulfill the inclusion criteria will be invited to the Stockholm University Brain Imaging Centre (SUBIC), where they will perform fMRI testing, tests of arithmetic, working memory and non-verbal intelligence.

\section{Brain imaging and analyses}

\section{Stimulus material}

The material will consist of four experimental and one baseline condition; simple multiplication (operands < 10, e.g. $03 \times 05=15$ ), difficult multiplication (one operand > 10, e.g. $22 \times 03=66$ ), simple subtraction (all numbers $<10$, e.g. $08-06=02$ ), difficult subtraction (answers $>10$ and with borrowing effect, e.g. $41-8=33$ ) and baseline (same digits, e.g. $03=03=03$ ). The task will be to identify, by button press if the stated equation is correct or not (correct in the baseline task refers to the same digit at all places, e.g. $03=03=03$ is correct, but $03=07=03$ is incorrect). The distance between proposed and correct answer is balanced over conditions and varies between -3 to +3 from the correct answer in all conditions.

There will be 64 unique trials for each condition. For all conditions, the proportion of targets and foils will be 1:1 (32 with correct and 32 with incorrect answers). Blocks with performance below $50 \%$ will be excluded from further analyses. Using 0 before single digits will equalize the visual appearance of the stimulus, leading to comparable activation of visual brain regions. Behavioral pilot testing of the material has shown that although the participants experience the 0:s before the number (e.g. 03) as distracting it does not interfere with accuracy or response time. 
The tasks of interest are the arithmetic tasks. The baseline condition will only be used, during fMRI analysis, to subtract processes common for all tasks, i.e. visual input as well as button-press, such that the fMRI analysis is focused on task-specific activation. (Sometimes it turns out that the baseline task is not as optimal as has been anticipated. If we will experience problems with the baseline task we will be able to contrast the other tasks to rest instead.)

The participants will practice the tasks off-line before entering the scanner, with materials not used in the scanner. In addition to the fMRI data, behavioral data from the button pressing will be collected and analyzed. Stimuli will be presented using the Psychtoolbox running under MatLab 2019a (The MathWorks Inc., Natick, MA).

\section{Experimental design}

The experiment is set up as a $2 \times 2 \times 2$ factorial blocked design with group (deaf, hearing) as betweensubject factor and type and difficulty as within-subject factors, where 'type' refers to subtraction or multiplication and 'difficulty' to simple or difficult operations. The blocked design will include two runs with a total of sixty-four trials for each of the five conditions. There will be eight trials in each block and four blocks of each type per run. During each trial, the stimulus will be displayed for 3000 $\mathrm{ms}$ with $50 \mathrm{~ms}$ intertrial-pause, such that each block lasts for $24350 \mathrm{~ms}$. To guard against differences in visual stimulation, the stimuli will be visible during the $3000 \mathrm{~ms}$ regardless of when the answer is given. Button press with the index and middle finger will be used.

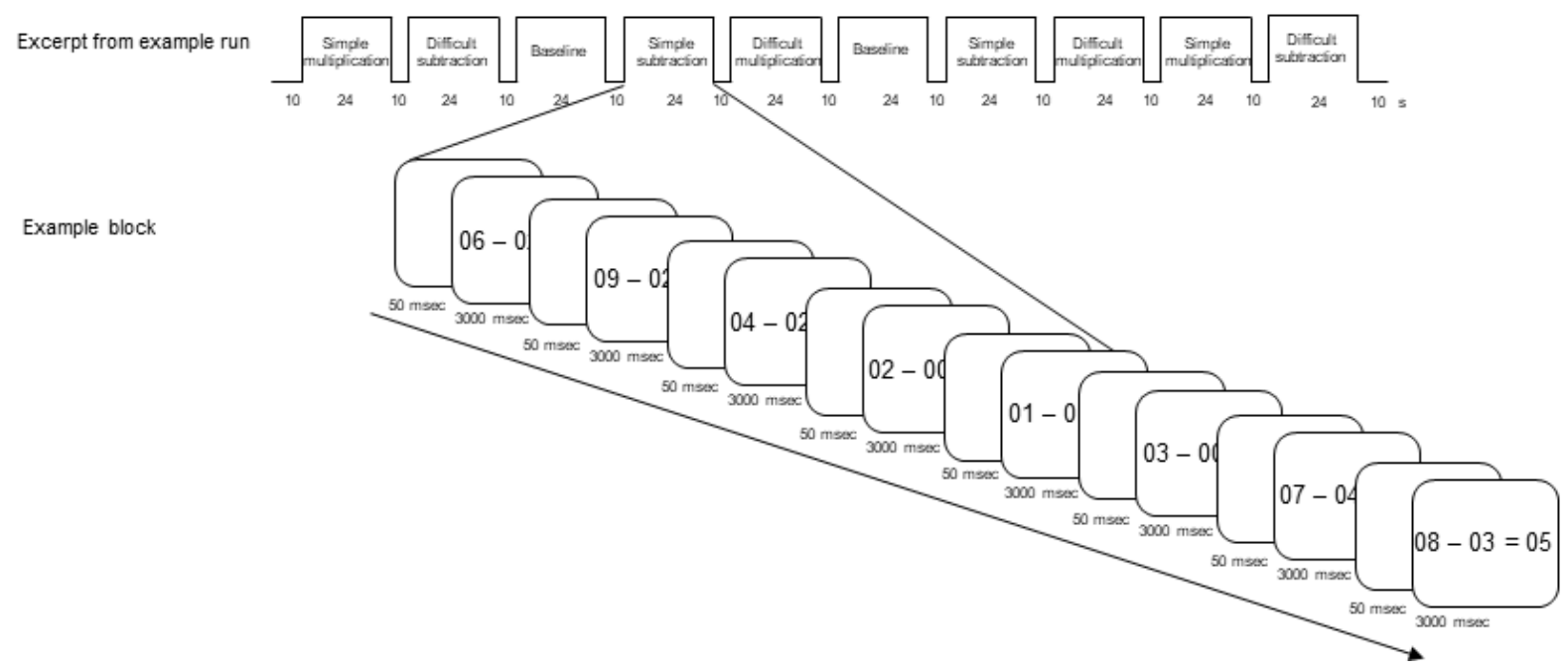

Figure 2. Schematic representation of an excerpt from a scanning run and an example of stimulus display and timing within a block of simple subtraction.

The between-block interval will be 10000 ms. Each run will start with a blank screen for approximately 10 seconds to allow for magnetization to stabilize to steady-state and end with a blank screen for approximately 10 seconds to capture the hemodynamic response of the last block. Hence, the experiment will consist of two approximately 12 minute long runs. An overview of the design is shown in figure 2. Within each task type, the 64 different trials have been pseudo-randomized into eight blocks, in which the proportion of correct to incorrect trials is between 3:5 to 5:3 (i.e. 3, 4 or 5 correct trials in each block, to prevent expectation effects). The blocks will be presented pseudorandomly and permuted within each run, such that every block type is equally likely to appear as starting block in a run and such that every block type is presented once within each epoch (i.e. all block types will appear once before any block type is repeated within each run). There will be as many unique compositions of blocks during the two runs as there are participants in one group, i.e. 
the same block order will be used for the first participant in both groups, a new order will be used for the second participant in both groups, etc. To further avoid order effects there will always be a block order for one participant (of each group) that is the reverse of the block order of another participant (i.e. participant no 2 in each group will be given the reverse block order compared to participant no 1 in each group, participant no 4 in each group will be given the reverse of participant no 3 in each group). In total, all participants will perform the same 320 trials (64 trials for each of the five task types) but in different orders.

After the two runs of arithmetic, included in this study, the participants will also perform two runs of geometry tasks. The geometry part will make a separate exploratory publication.

\section{Data acquisition}

MRI data will be acquired on a 3.0 T scanner (Siemens Magnetom Prisma, Siemens Healthcare $\mathrm{GmbH}$ ), equipped with a 64-channel head coil, at Stockholm University Brain Imaging Center (Stockholm, Sweden: SUBIC). Functional data will be collected, during continuous scanning, using a BOLD EPI sequence (TE/TR = 30/1760 ms, FA = 70으, oblique axial slice orientation, FOV = $192 \times 192$ $\mathrm{mm}$, slice thickness $=2$, in-plane resolution $=2 \times 2 \mathrm{~mm}$, number of slices $=58$, GRAPPA acc. $=2$, SMS acc. $=2$ ). We will also collect T1-weighted structural image (3D MPRAGE, TI/TE/TR =900/2.98/2300 ms, FA = 9o, oblique axial slice orientation, $\mathrm{FOV}=256 \times 256 \mathrm{~mm}, 256 \times 256 \times 208$ acquisition matrix, $1 \times 1$ $x 1 \mathrm{~mm}^{3}$ voxels, GRAPPA acc. factor $=2$, scan time $=5: 21$ ) that will be used for normalizing the functional data to MNI space.

In the case of incidental findings during scanning, these will be referred to the radiologist engaged by Stockholm Brain University Imaging Centre. The radiologist will examine the findings and if necessary, refer the participant to a primary care physician for further medical examination. Before scanning, participants will be informed about what incidental finding is and how such findings will be handled.

\section{Statistical analyses of imaging data}

Preprocessing and analysis will be performed using statistical parametric mapping packages (SPM12 or higher; Wellcome Trust Centre for Neuroimaging, London, UK) and CONN toolbox (www.nitrc.org/projects/conn, Whitfield-Gabrieli \& Nieto-Castanon, 2012) running under MatLab R2019a (The MathWorks Inc., Natick, MA).

Preprocessing. Before SPM-preprocessing, the Dicom-images from the MR-scanner will be converted to NIFTI format using the Dicom-to-nifti converter in MRIcron (Rorden \& Brett, 2000). Preprocessing will be performed following standard SPM12 procedure and include 1) realignment, which is performed to correct for subject motion between volumes, 2) coregistration of the structural and functional images, 3) segmentation and spatial normalization to match the geometry of subject brain to standard space, 4) normalization of functional and structural images where a segmented deformation field from the previous step is applied to all functional and structural images and 5) spatial smoothing to increase sensitivity using an $8 \mathrm{~mm}$ FWHM Gaussian kernel. Individuals with more than $3 \mathrm{~mm}$ movement in $\mathrm{x}, \mathrm{y}, \mathrm{z}$ or 3 degrees in pitch, yaw, roll, will be excluded or partially excluded (i.e. if movement is restricted to one run, the other run will be included in the analyses).

To investigate the primary objective of this study (prediction 1-4) region of interest (ROI) and connectivity analyses will be performed. The secondary exploratory objective (prediction 5-7) will be tested using whole-brain analyses.

Whole-brain analyses. Whole-brain analyses will be conducted by fitting a general linear model with regressors representing the five different task types as well as the six motion parameters derived from the realignment procedure. At first level, individual statistical parametric map images 
pertaining to each of the four experimental tasks will be contrasted with the baseline task. These images will, individually for each participant, be analyzed through a $2 \times 2$ [type $x$ difficulty] ANOVA. From the ANOVA, contrast files pertaining to the main effect of type and difficulty as well as the interaction effect between type and difficulty will be brought into second-level analyses where onesample t-test will be performed separately for deaf signers and hearing non-signers. Finally, secondlevel between-group analyses will be performed by bringing the individual contrast files into onesample t-tests for the main effect of type and difficulty and in two-sample t-tests for the main effect of group and interaction effects. To investigate the nature of significant interaction effects beta values from the peak coordinate will be extracted for each of the ingoing contrast (i.e. an interaction between type and difficulty will be investigated by extracting beta values for simple and difficult subtraction and simple and difficult multiplication).

Because we expect similar activation for both groups, at least for difficult multiplication (see Fig. 1a), conjunction analyses for type and difficulty across groups will be performed to answer questions about similarities in activation between groups. Significant conjunction indicates that the contrasts evaluated are consistently high and jointly significant across the tested conditions (Friston, Penny, \& Glaser, 2005).

Activation will be considered as significant if $p_{f w e}<.05$ at peak level. Because brain activation data from deaf individuals are known to be very heterogeneous, it can be difficult to obtain activation that survives the conservative family-wise error correction at peak level. If this will be the case here, cluster-level analyses at $p<.005$ will also be reported.

Region of interest analyses. To investigate the specific hypotheses, ROI-analyses will be performed. Region of interests will be the anterior and posterior portion of the left angular gyrus, the whole angular gyrus, right horizontal intraparietal sulcus, the left inferior frontal gyrus and bilateral hippocampus as defined by the SPM Anatomy Toolbox (version 2.2b or later; Eickhoff et al., 2005). Since the literature on the angular gyrus is diverging, we will analyze both anterior, posterior and the whole angular gyrus. ROI mean values will be obtained for each contrast (task minus baseline, as described above under "whole-brain analyses") from each ROI for every individual, again using the SPM Anatomy Toolbox. Mean values will then be entered into a $2 \times 2 \times 2$ (type $x$ difficulty $\times$ group) ANOVA in SPSS statistics 25 (IBM, SPSS Statistics, version 25, IBM Corporation, New York, USA).

Connectivity analyses. Task-based connectivity will be performed using Conn - functional connectivity toolbox (version 17, or later; www.nitrc.org/projects/conn, RRID:SCR_009550).

Correlational analyses between the BOLD signal in the seed ROI's and all ROI's and networks included in Conn will be performed to obtain ROI-to-ROI connectivity estimations for each participant. The seed ROI's will be the same as in the ROI-analyses described above, i.e. ROI's from the anatomy toolbox will be entered as seed-ROI's in Conn. This choice is because the ROI-s available in Conn do not include the subdivision of angular gyrus. First-level covariates will include realignment parameters. Second-level covariates will be group (deaf/hearing), age and raw score from the nonverbal intelligence test. Denoising, including using standard Conn recommendations, will be carried out to remove unwanted motion and artifacts from the BOLD signal before connectivity measures are computed. High-pass filtering at $0.008 \mathrm{~Hz}$ will also be applied. At second-level, within-group connectivity, as well as between-group connectivity, will be analyzed for the four tasks.

\section{Behavioral tests and analyses}

Behavioral measures of working memory, arithmetic skill, and in-scanner performance will be collected and used to ensure that potential group differences in brain activation are not due to 
differences in these skills. All behavioral data will be analyzed using SPSS statistics 25 (IBM, SPSS Statistics, version 25, IBM Corporation, New York, USA).

\section{Working memory}

To assess the participant's working memory ability, we will conduct a computerized version of the Corsi block-tapping test (Corsi, 1972). In this test, nine colored squares will appear on the screen. A sequence of blocks will change color to another color for $500 \mathrm{~ms}$ (inter-stimulus interval $500 \mathrm{~ms}$ ). After the last block in the sequence has been lit up a signal will be given that informs the participant that they should start clicking, using a computer mouse, at the squares in the same order. In the first sequence, two squares will be included in the sequence. If the participant correctly reproduces the sequence the next sequence will increase by one. If the participant fails to reproduce the sequence, they will be given a second attempt at the same sequence length. The test ends after two attempts at which the participant fails to reproduce a sequence. The span length is defined by the number of squares include in the last sequence the participant reproduces correctly. The test will be implemented in Psychtoolbox running under MatLab 2019a (The MathWorks Inc., Natick, MA).

\section{Arithmetic skills}

To ensure and control for differences in performance between groups, arithmetic skills will be tested using a computerized version of the Skagerlund arithmetic test (Skagerlund et al., 2019). The test includes four subtests, one for each operation type (addition, subtraction, multiplication, and division). In each subtest, the participant will be asked to complete as many arithmetic problems as possible within 120 seconds. The difficulty of the problems increases within each subtest by increasing the number of digits included or by requiring borrowing and carrying. Each subtest includes 54 problems, except for the division subtest that contained 27 problems. The total score is the number of correctly completed problems, with a maximum score of 189 . The test will be implemented in Psychtoolbox running under MatLab 2019a (The MathWorks Inc., Natick, MA). Results will be analyzed as a $2 \times 4$ ANOVA with group (deaf, hearing) as between-group factor and operation type (addition, subtraction, multiplication, division) as within-subject factor. Results from this test will be important in order to interpret imaging results. For example, if deaf individuals perform as fast as hearing in multiplication but still engages the right intraparietal sulcus it would indicate qualitatively different processes. (The implementation in Psychtoolbox makes it possible to analyze specific items. These analyses are not planned. Hence, if necessary, they will be added in a post-hoc-section after all planned tests.)

\section{In-scanner responses}

Results from the in-scanner performance will be analyzed to assess potential differences between groups and between the different operation types and difficulty levels. Response time and accuracy till be analyzed in two separate mixed $2 \times 2 \times 2$ analyses of variance with group (deaf or hearing) as between-subject factor and type (subtraction or multiplication) and difficulty (simple or difficult) as within-subject factors.

\section{Timeline}

June 2019

First pilot was performed in the scanner. Size of presented stimuli, choice of response button pad and decisions on coil type were decided upon. Problems with a new button pad call for a second pilot. 
September 2019

Autumn 2019

December 2020

Spring 2021

June 2021
Second pilot in the was performed. Problems from the first pilot have now been solved.

Start of data collection.

Data collection will end.

Analyses and writing up results.

Submission for Stage 2 review.

\section{Conflict of Interest}

No conflict of interest to declare.

\section{References}

Andin, J., Fransson, P., Dahlström, Ö., Rönnberg, J., \& Rudner, M. (2019). The neural basis of arithmetic and phonology in deaf signing individuals. Language, Cognition and Neuroscience, 1-13. doi:10.1080/23273798.2019.1616103

Andin, J., Fransson, P., Ronnberg, J., \& Rudner, M. (2018). fMRI Evidence of Magnitude Manipulation during Numerical Order Processing in Congenitally Deaf Signers. Neural Plasticity, 2018, 2576047. doi:10.1155/2018/2576047

Andin, J., Ronnberg, J., \& Rudner, M. (2014). Deaf signers use phonology to do arithmetic. Learning and Individual Differences, 32, 246-253. doi:10.1016/j.lindif.2014.03.015

Arsalidou, M., Pawliw-Levac, M., Sadeghi, M., \& Pascual-Leone, J. (2018). Brain areas associated with numbers and calculations in children: Meta-analyses of fMRI studies. Dev Cogn Neurosci, 30, 239-250. doi:10.1016/j.dcn.2017.08.002

Arsalidou, M., \& Taylor, M. J. (2011). Is 2+2=4? Meta-analyses of brain areas needed for numbers and calculations. Neuroimage, 54(3), 2382-2393. doi:10.1016/j.neuroimage.2010.10.009

Artemenko, C., Soltanlou, M., Bieck, S. M., Ehlis, A.-C., Dresler, T., \& Nuerk, H.-C. (2019). Individual Differences in Math Ability Determine Neurocognitive Processing of Arithmetic Complexity: A Combined fNIRS-EEG Study. Frontiers in Human Neuroscience, 13(227). doi:10.3389/fnhum.2019.00227

Artemenko, C., Soltanlou, M., Dresler, T., Ehlis, A. C., \& Nuerk, H. C. (2018). The neural correlates of arithmetic difficulty depend on mathematical ability: evidence from combined fNIRS and ERP. Brain Structure \& Function, 223(6), 2561-2574. doi:10.1007/s00429-018-1618-0

Bagga-Gupta, S. (2004). Literacy and deaf education - A theoretical analysis of the international and Swedish literature. Stockholm.

Bloechle, J., Huber, S., Bahnmueller, J., Rennig, J., Willmes, K., Cavdaroglu, S., . . Klein, E. (2016). Fact learning in complex arithmetic-the role of the angular gyrus revisited. Hum Brain Mapp, 37(9), 3061-3079. doi:10.1002/hbm.23226

Bull, R., Blatto-Vallee, G., \& Fabich, M. (2006). Subitizing, magnitude representation, and magnitude retrieval in deaf and hearing adults. J Deaf Stud Deaf Educ, 11(3), 289-302. doi:10.1093/deafed/enj038

Bull, R., Marschark, M., \& Blatto-Vallee, G. (2005). SNARC hunting: Examining number representation in deaf students. Learning and Individual Differences, 15(3), 223-236. doi:10.1016/j.lindif.2005.01.004

Cardin, V., Rudner, M., De Oliveira, R. F., Andin, J., Su, M. T., Beese, L., . . Ronnberg, J. (2018). The Organization of Working Memory Networks is Shaped by Early Sensory Experience. Cereb Cortex, 28(10), 3540-3554. doi:10.1093/cercor/bhx222 
Corsi, P. M. (1972). Human mamory and the medial temporal region of the brain. (Doctoral dissertation), McGill University, Montreal. (Daneman, M., Carpenter, P. University Mocrofilms No AA105-77717. Abstracts International. 34:819B)

Dehaene, S. (1992). Varieties of numerical abilities. Cognition, 44(1-2), 1-42.

Dehaene, S., Piazza, M., Pinel, P., \& Cohen, L. (2003). Three parietal circuits for number processing. Cognitive Neuropsychology, 20(3-6), 487-506. doi:10.1080/02643290244000239

Eichenbaum, H. (2004). Hippocampus: cognitive processes and neural representations that underlie declarative memory. Neuron, 44(1), 109-120. doi:10.1016/j.neuron.2004.08.028

Eickhoff, S. B., Stephan, K. E., Mohlberg, H., Grefkes, C., Fink, G. R., Amunts, K., \& Zilles, K. (2005). A new SPM toolbox for combining probabilistic cytoarchitectonic maps and functional imaging data. Neuroimage, 25(4), 1325-1335. doi:10.1016/j.neuroimage.2004.12.034

Fedorenko, E., Duncan, J., \& Kanwisher, N. (2012). Language-Selective and Domain-General Regions Lie Side by Side within Broca's Area. Current Biology, 22(21), 2059-2062. doi:https://doi.org/10.1016/i.cub.2012.09.011

Friston, K. J., Penny, W. D., \& Glaser, D. E. (2005). Conjunction revisited. Neuroimage, 25(3), 661-667. doi:10.1016/j.neuroimage.2005.01.013

Ganor-Stern, D. (2016). Solving Math Problems Approximately: A Developmental Perspective. PLoS One, 11(5), e0155515. doi:10.1371/journal.pone.0155515

Grabner, R. H., Ansari, D., Koschutnig, K., Reishofer, G., \& Ebner, F. (2013). The function of the left angular gyrus in mental arithmetic: evidence from the associative confusion effect. Hum Brain Mapp, 34(5), 1013-1024. doi:10.1002/hbm.21489

Grabner, R. H., Ansari, D., Koschutnig, K., Reishofer, G., Ebner, F., \& Neuper, C. (2009). To retrieve or to calculate? Left angular gyrus mediates the retrieval of arithmetic facts during problem solving. Neuropsychologia, 47(2), 604-608. doi:10.1016/j.neuropsychologia.2008.10.013

Grabner, R. H., Ansari, D., Reishofer, G., Stern, E., Ebner, F., \& Neuper, C. (2007). Individual differences in mathematical competence predict parietal brain activation during mental calculation. Neuroimage, 38(2), 346-356. doi:10.1016/j.neuroimage.2007.07.041

Grabner, R. H., Reishofer, G., Koschutnig, K., \& Ebner, F. (2011). Brain correlates of mathematical competence in processing mathematical representations. Frontiers in Human Neuroscience, 5, 130. doi:10.3389/fnhum.2011.00130

Hirshorn, E. A., Dye, M. W., Hauser, P. C., Supalla, T. R., \& Bavelier, D. (2014). Neural networks mediating sentence reading in the deaf. Frontiers in Human Neuroscience, 8, 394. doi:10.3389/fnhum.2014.00394

Hyde, M., Zevenbergen, R., \& Power, D. (2003). Deaf and hard of hearing students' performance on arithmetic word problems. Am Ann Deaf, 148(1), 56-64.

Jolles, D., Ashkenazi, S., Kochalka, J., Evans, T., Richardson, J., Rosenberg-Lee, M., . . Menon, V. (2016). Parietal hyper-connectivity, aberrant brain organization, and circuit-based biomarkers in children with mathematical disabilities. Dev Sci, 19(4), 613-631. doi:10.1111/desc.12399

Kelly, R. R., Lang, H. G., Mousley, K., \& Davis, S. M. (2003). Deaf college students' comprehension of relational language in arithmetic compare problems. J Deaf Stud Deaf Educ, 8(2), 120-132. doi:10.1093/deafed/eng0068/2/120 [pii]

Klein, E., Suchan, J., Moeller, K., Karnath, H.-O., Knops, A., Wood, G., . . Willmes, K. (2016). Considering structural connectivity in the triple code model of numerical cognition: differential connectivity for magnitude processing and arithmetic facts. Brain Structure and Function, 221(2), 979-995. doi:10.1007/s00429-014-0951-1

Klein, E., Willmes, K., Bieck, S. M., Bloechle, J., \& Moeller, K. (2019). White matter neuro-plasticity in mental arithmetic: Changes in hippocampal connectivity following arithmetic drill training. Cortex, 114, 115-123. doi:10.1016/j.cortex.2018.05.017

Lee, K. M., \& Kang, S. Y. (2002). Arithmetic operation and working memory: differential suppression in dual tasks. Cognition, 83(3), B63-68. doi:doi.org/10.1016/S0010-0277(02)00010-0 
LGr 80. (1983). Läroplan för grundskolan [Curriculum for elementary school]. Stockholm: Liber Utbildningsförlaget.

Malaia, E., Talavage, T. M., \& Wilbur, R. B. (2014). Functional connectivity in task-negative network of the Deaf: effects of sign language experience. PeerJ, 2, e446. doi:10.7717/peerj.446

Masataka, N., Ohnishi, T., Imabayashi, E., Hirakata, M., \& Matsuda, H. (2006). Neural correlates for numerical processing in the manual mode. J Deaf Stud Deaf Educ, 11(2), 144-152. doi:10.1093/deafed/enj017

Mayberry, R. I., \& Lock, E. (2003). Age constraints on first versus second language acquisition: evidence for linguistic plasticity and epigenesis. Brain Lang, 87(3), 369-384. doi:S0093934X03001378 [pii]

Mumford, J. A., \& Nichols, T. E. (2008). Power calculation for group fMRI studies accounting for arbitrary design and temporal autocorrelation. Neuroimage, 39(1), 261-268. doi:10.1016/j.neuroimage.2007.07.061

Nunes, T., Bryant, P., Burman, D., Bell, D., Evans, D., \& Hallett, D. (2009). Deaf children's informal knowledge of multiplicative reasoning. J Deaf Stud Deaf Educ, 14(2), 260-277. doi:10.1093/deafed/enn040

Pagliaro, C. M. (2010). Mathematics instruction and learning of deaf and hard-of-hearing students: What do we know? Where do we go? . In P. Nathan, M. Marschark, \& S. P.E. (Eds.), The Oxford handbook of deaf studies, language and education (Vol. 2, pp. 156-171). New York: Oxford university press.

Prado, J., Mutreja, R., Zhang, H., Mehta, R., Desroches, A. S., Minas, J. E., \& Booth, J. R. (2011). Distinct representations of subtraction and multiplication in the neural systems for numerosity and language. Hum Brain Mapp, 32(11), 1932-1947. doi:10.1002/hbm.21159

Price, G. R., \& Ansari, D. (2011). Symbol processing in the left angular gyrus: evidence from passive perception of digits. Neuroimage, 57(3), 1205-1211. doi:10.1016/j.neuroimage.2011.05.035

Roos, C. (2006). Teckenspråk och pedagogik [Sign language and pedagogy]. (SOU 2006:29). Stockholm, Sweden.

Rorden, C., \& Brett, M. (2000). Stereotaxic display of brain lesions. Behav Neurol, 12(4), 191-200.

Rosenberg-Lee, M., Ashkenazi, S., Chen, T., Young, C. B., Geary, D. C., \& Menon, V. (2015). Brain hyper-connectivity and operation-specific deficits during arithmetic problem solving in children with developmental dyscalculia. Dev Sci, 18(3), 351-372. doi:10.1111/desc.12216

Sandler, W., \& Lillo-Martin, D. (2006). Sign language and linguistic universals. Cambridge: Cambridge university press.

Serrano Pau, C. (1995). The deaf child and solving problems of arithmetic. The importance of comprehensive reading. American Annals of the Deaf, 140(3), 287-290. doi:10.1353/aad.2012.0599

Simon, O., Mangin, J. F., Cohen, L., Le Bihan, D., \& Dehaene, S. (2002). Topographical layout of hand, eye, calculation, and language-related areas in the human parietal lobe. Neuron, 33(3), 475487. doi:Doi 10.1016/S0896-6273(02)00575-5

Skagenholt, M., Träff, U., Västfjäll, D., \& Skagerlund, K. (2018). Examining the Triple Code Model in numerical cognition: An fMRI study. PLoS One, 13(6), e0199247-e0199247. doi:10.1371/journal.pone.0199247

Skagerlund, K., Bolt, T., Nomi, J. S., Skagenholt, M., Vastfjall, D., Traff, U., \& Uddin, L. Q. (2019). Disentangling Mathematics from Executive Functions by Investigating Unique Functional Connectivity Patterns Predictive of Mathematics Ability. J Cogn Neurosci, 31(4), 560-573. doi:10.1162/jocn_a_01367

Sokolowski, H. M., Fias, W., Mousa, A., \& Ansari, D. (2017). Common and distinct brain regions in both parietal and frontal cortex support symbolic and nonsymbolic number processing in humans: A functional neuroimaging meta-analysis. Neuroimage, 146, 376-394. doi:10.1016/j.neuroimage.2016.10.028

Soltanlou, M., Artemenko, C., Dresler, T., Haeussinger, F. B., Fallgatter, A. J., Ehlis, A. C., \& Nuerk, H. C. (2017). Increased arithmetic complexity is associated with domain-general but not 
domain-specific magnitude processing in children: A simultaneous fNIRS-EEG study. Cogn Affect Behav Neurosci, 17(4), 724-736. doi:10.3758/s13415-017-0508-x

Titus, J. C. (1995). The concept of fractional number among deaf and hard of hearing students. Am Ann Deaf, 140(3), 255-262.

Twomey, T., Waters, D., Price, C. J., Evans, S., \& MacSweeney, M. (2017). How Auditory Experience Differentially Influences the Function of Left and Right Superior Temporal Cortices. J Neurosci, 37(39), 9564-9573. doi:10.1523/jneurosci.0846-17.2017

Uddin, L. Q., Supekar, K., Amin, H., Rykhlevskaia, E., Nguyen, D. A., Greicius, M. D., \& Menon, V. (2010). Dissociable connectivity within human angular gyrus and intraparietal sulcus: evidence from functional and structural connectivity. Cereb Cortex, 20(11), 2636-2646. doi:10.1093/cercor/bhq011

Whitfield-Gabrieli, S., \& Nieto-Castanon, A. (2012). Conn: a functional connectivity toolbox for correlated and anticorrelated brain networks. Brain Connectivity, 2(3), 125-141. doi:10.1089/brain.2012.0073

Wu, S. S., Chang, T. T., Majid, A., Caspers, S., Eickhoff, S. B., \& Menon, V. (2009). Functional heterogeneity of inferior parietal cortex during mathematical cognition assessed with cytoarchitectonic probability maps. Cereb Cortex, 19(12), 2930-2945. doi:10.1093/cercor/bhp063

Zhou, X., Li, M., Li, L., Zhang, Y., Cui, J., Liu, J., \& Chen, C. (2018). The semantic system is involved in mathematical problem solving. Neuroimage, 166, 360-370.

doi:10.1016/j.neuroimage.2017.11.017 